\title{
Cooperative Spectrum Sharing Protocol Based on Transform Domain Processing with Joint Secondary Selection and Power Allocation
}

\author{
Xuanli Wu, Xingling Han \\ School of Electronic and Information Engineering \\ Harbin Institute of Technology, Harbin, China \\ xlwu2002@hit.edu.cn, hanxingling1993@126.com
}

\author{
Fabrice Labeau \\ Department of Electrical and Computer Engineering, \\ McGill University, Quebec, Canada \\ fabrice.labeau@mcgill.ca
}

\begin{abstract}
In order to reduce interference to the primary system and further improve the spectrum efficiency, the Fractional Fourier Transform is introduced as a tool to solve the spectrum sharing problem and a new spectrum sharing protocol is proposed in this paper where sinusoidal signals and chirp signals are utilized for primary users and cognitive users, respectively. In our spectrum sharing protocol, secondary users take the initiative to access the spectrum or act as a DF relay to assist the primary system adaptively according to the channel conditions of the direct transmission link. Moreover, the joint optimization of power allocation and user selection of distributed secondary users is studied to achieve the maximum achievable data rate for the secondary system while guaranteeing the target data rate of primary user. Simulation results show that compared with previous protocols, the outage probability of both primary and secondary systems can be improved, and with an increase in the number of secondary users, a higher achievable data rate can be obtained for the secondary system.
\end{abstract}

Keywords: cooperative spectrum sharing; Fractional Fourier Transform; user selection; power allocation

\section{INTRODUCTION}

In order to adequately utilize spectrum resources and effectively improve spectrum efficiency, Cognitive Radio (CR) technology has been proposed to exploit underutilized spectrum in an opportunistic manner [1]. In traditional Cognitive Radio systems, secondary users can only access the spectrum in the absence of primary users and thus it is difficult for secondary users to realize continuous reliable communications. Hence, the spectrum sharing technology, aiming to achieve efficient and reliable communications of secondary users while guaranteeing the quality of service (QoS) of primary users, has attracted more attention [2-3].

Since the performance of communications can be greatly improved through the cooperation between primary users and secondary users, cooperative spectrum sharing problem has been studied extensively. In [4], a two-phase spectrum sharing protocol employing Amplify and Forward (AF) mechanism is proposed where the primary system allocates a fraction of time and power to the secondary system in exchange for cooperation. However, the performance of this protocol is not satisfactory due to the fact that noise accumulates with AF relaying. In order to solve this problem, a two-phase protocol based on Decode and Forward (DF) relaying is given in [5], where secondary users can access the spectrum by applying as a relay to transmit the linear combination of the primary user's signal and its own secondary signal. However, due to the fact that this composite signal will induce interference to both primary and secondary system, the outage probability of such DF based protocol is far from optimal. Moreover, this protocol has a stringent restriction on the distance between primary user and secondary user.

In the following study, in order to reduce the interference to primary users, ref. [6] proposes a cooperative spectrum sharing protocol with joint time and power allocation. Then, the cooperative spectrum sharing protocols in which the secondary user cooperates to help the primary user reach its target data rate with a fraction of power and subcarriers or bandwidth while using the remaining power and subcarriers or bandwidth to transmit its own signal is proposed in [7-8]. However, the above-mentioned works focus on spectrum sharing adopting the strategy that the secondary user uses disjoint time or bandwidth to forward the signal of the primary user and transmit the signal of the secondary user. However, the spectrum cannot be fully utilized in this way. In addition, a spectrum sharing strategy which adopts multiple antenna technology to distinguish the primary signal and secondary signal has been proposed [9]. Although the strategy can guarantee the coexistence of primary and secondary users in the licensed channel and satisfy their requirements of communication, excessive computational complexity makes it difficult to be applied.

In this paper, in order to further improve the spectrum utilization and reduce interference to the primary system effectively, we introduce the application of the Fractional Fourier Transform (FrFT) technology to spectrum sharing and we propose a new cooperative spectrum sharing protocol. In our proposed protocol, sinusoidal signals and chirp signals occupying the same frequency band are applied to primary and secondary users, respectively. Due to the energy concentration property of chirp signals in the FrFT domain, a chirp signal can be easily filtered out from a sinusoidal signal 
in the FrFT domain [10]. Moreover, most existing spectrum sharing protocols just consider the single case where the direct transmission link of the primary user is good enough to tolerate additional interference or too bad so that assistance is needed. In our proposed spectrum sharing protocol, different channel conditions for the direct transmission link of the primary user (PU) are considered. When the direct transmission link is good enough to support the PU's target data rate requirement, the secondary user can actively access the frequency spectrum without affecting the primary user's requirement by using power control. When the direct transmission link is not of sufficient quality to guarantee the PU's QoS requirement, the secondary users can act as DF relays with a fraction of power to assist the primary user. As a reward, secondary users can utilize the remaining power to transmit their own secondary signal. In this way, system spectrum resource can be utilized adequately.

Then we study the joint optimization of power allocation and user selection of distributed secondary users such that the transmission data rate of the secondary user (SU) can be maximized subject to the target data rate constraint of primary user. The influence of the number of secondary users and transmit power on the performance of primary and secondary systems is analyzed. Simulation results show that the efficiency of our proposed spectrum sharing protocol as well as the outage probability of both primary and secondary systems can be improved significantly, and the achievable data rate of the secondary system can also be improved with the increase of the number of secondary users.

The remainder of this paper is organized as follows: Section II describes the system model. Detailed analysis of our proposed cooperative spectrum sharing protocol with joint user selection and power allocation is given in Section III. In Section IV, the performance comparison between the proposed spectrum sharing protocol and the protocol in [11] is performed. Finally, conclusions are drawn in Section V.

\section{SYSTEM MODEL}

In this paper, we consider an overlay cognitive radio network. The primary system comprises a pair of primary transmitter PT and receiver PR, denoted as terminal 1 and terminal 2, respectively, as shown in Fig 1. The secondary system contains $N$ secondary transmitter $\mathrm{ST}_{n}$, denoted as terminal $3_{n}, n \in\{1,2 \ldots N\}$, and one receiver SR denoted as terminal 4. It is assumed that the channel coefficient over links is denoted as $h_{i j}=\sqrt{d_{i j}^{-v}} g_{i j}$, where $v$ is the path loss coefficient, $d_{i j}$ is the normalized distance from terminal $i$ to terminal $j$ and $g_{i j}$ is the fading coefficient from terminal $i$ to terminal $j$ which is modeled as a Rayleigh variable. Thus $\left|g_{i j}\right|^{2}$ is exponentially distributed with mean $1 / \delta_{i j}$, i.e., $\left|g_{i j}\right|^{2} \sim \varepsilon\left(\delta_{i j}\right)$. Hence, $\left|h_{i j}\right|^{2}$ obeys exponential distribution with mean $1 / d_{i j}^{v} \delta_{i j}$, i.e., $\left|h_{i j}\right|^{2} \sim \varepsilon\left(d_{i j}^{v} \delta_{i j}\right)$ [12]. In this paper, we assume that the primary receiver PR can obtain the instantaneous channel gain of direct transmission link PT $\rightarrow$ PR and the secondary transmitter $\mathrm{ST}_{n}$ can obtain the instantaneous channel gain of their related transmission links $\mathrm{PT} \rightarrow \mathrm{ST}_{n}, \mathrm{ST}_{n} \rightarrow \mathrm{PR}$ and $\mathrm{ST}_{n} \rightarrow \mathrm{SR}(n=1,2 . . N)$.

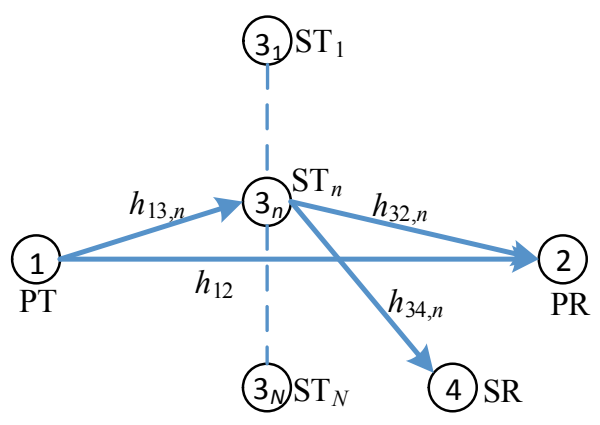

Fig.1 System model

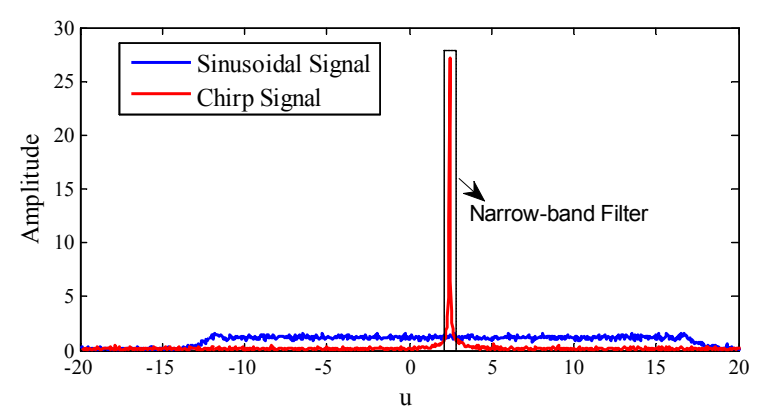

Fig.2 Sinusoidal signal and chirp signal in FrFT domain

Thanks to the energy concentration property of chirp signals in the FrFT domain, chirp signals can be easily separated from sinusoidal signals. Hence, we use sinusoidal signal and chirp signals for primary user and secondary users, respectively. By properly designing the parameters of sinusoidal and chirp signals, the sinusoidal signals can show flat distribution in the FrFT domain so that the chirp signal can be easily filtered out in the FrFT domain with very narrow band filter. At the same time, very minor distortion can be found for sinusoidal signal after the filtering of chirp signal. The expression of these two kinds of signals can be expressed as: $\quad s(t)=A \times \cos \left(2 \pi f_{c} t+\varphi_{0}\right) \quad$ and $c(t)=A \times \cos \left(2 \pi f_{c} t+\varphi_{0}+k t^{2}\right)$, respectively, where $A$ is the amplitude of the signal, $f_{c}$ is the central frequency, $\varphi_{0}$ is the initial phase and $k$ is the chirp rate. The amplitude spectrum of these signals in the corresponding energy concentration FrFT domain is illustrated in Fig. 2.

\section{CoOperative SPECTRUM Sharing Protocol}

In this paper, a cooperative spectrum sharing protocol based on transform domain processing is proposed, and the transmission procedure for the proposed protocol is given in Fig.3. We assume that the primary and secondary nodes use a single antenna. The signals transmitted by primary user and secondary users are expressed as $x_{p}$ and $x_{s, n}(n=1,2 . . N)$, respectively, with the assumption that $E\left\{\left|x_{p}\right|^{2}\right\}=E\left\{\left|x_{s, n}\right|^{2}\right\}=1$. Since in each transmission slot, only one ST is selected to access the spectrum or assist the primary user, we remove the index of $n$ for simplification.

At the beginning of each transmission time slot, two cases for secondary spectrum access need to be considered depending on whether the Signal to Noise Ratio (SNR) $r_{12}$ of 


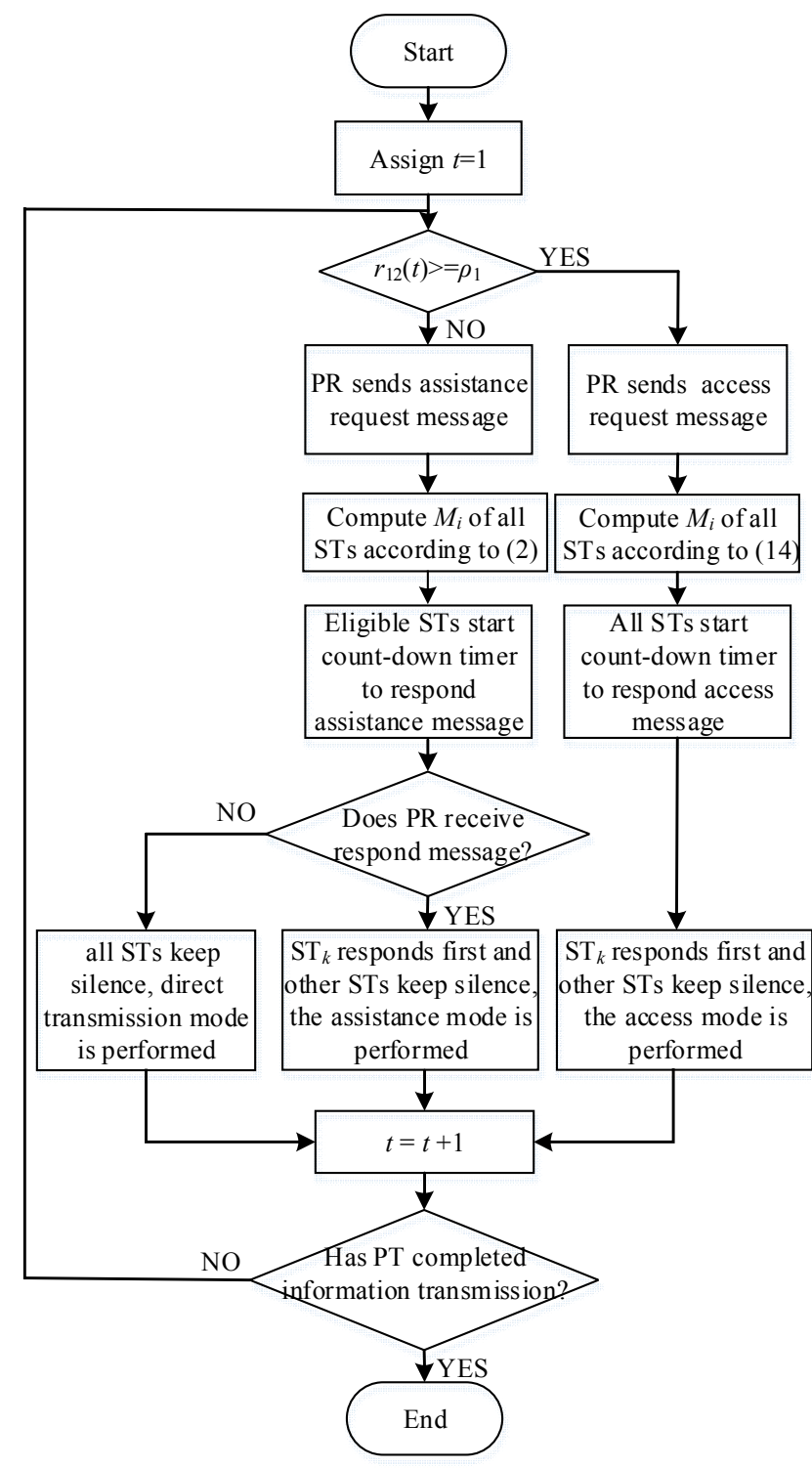

Fig.3 Flowchart of the proposed spectrum sharing protocol

primary direct transmission link is higher than a given threshold $\rho_{1}=2^{R_{p t}}-1$, where $R_{p t}$ is the target data rate of primary user. The SNR of direct link $r_{12}$ can be computed by:

$$
r_{12}=\left(P_{p}\left|h_{12}\right|^{2}\right) / \sigma_{w}^{2},
$$

where, $P_{p}$ is the transmit power of PT and $\sigma_{w}^{2}$ is the power of Gaussian noise.

Access Mode: when $r_{12}>\rho_{1}$ which indicates that the direct transmission link of primary system is good enough to achieve its target data rate $R_{p t}$, so that the primary system can tolerate additional interference caused by the secondary system. In this case, the primary receiver PR sends a spectrum sharing access request message which indicates the tolerable interference $I$. After listening to such message, $\mathrm{ST}_{i}$ computes its corresponding spectrum sharing priority $M_{i}$ with the consideration of channel gain of $\mathrm{ST}_{i} \rightarrow \mathrm{PR}$ links $\left|h_{32, i}\right|^{2}$ and
$\mathrm{ST}_{i} \rightarrow \mathrm{SR}$ links $\left|h_{34, i}\right|^{2} \quad(i=1 \sim N)$. The priority of each secondary user can be expressed as:

$$
M_{i}=\frac{\left|h_{34, i}\right|^{2}}{\exp \left(\left|h_{32, i}\right|^{2}\right)}, i=1,2 \ldots N .
$$

In (2), the priority of secondary user decreases rapidly with the channel gain of ST $\rightarrow$ PR link. In this way, the secondary with worse channel condition of ST $\rightarrow$ PR link have more opportunity to be selected and the interference due to the access of secondary user can be reduced. Moreover, the priority of secondary user increases with the channel gain of ST $\rightarrow$ SR link, so that the selected secondary user can transmit more data to $\mathrm{PR}$ while trying to reduce the interference to primary user.

In order to reduce the signaling overhead, similar to [11], each secondary user starts count-down timer with the initial value which is computed by:

$$
T_{i}=\left\lfloor\frac{\Gamma}{M_{i}}\right\rfloor, i=1,2 \ldots N,
$$

where, $\Gamma$ is a constant. From (3), the secondary user with the highest priority has the smallest initial value and thus the corresponding count-down timer decrease to zero first.

Assuming that $\mathrm{ST}_{k}$ 's timer decreases to zero first, it broadcasts the first access responding message. When the primary system receives the first access responding message, the corresponding secondary user will be selected and will work simultaneously with primary system.

In the access mode, the signal $x_{p}$ from PT and the signal $x_{s}$ from $\mathrm{ST}_{k}$ are transmitted simultaneously. The signals received by PR and SR are denoted as $y_{2}$ and $y_{4}$, respectively, and can be expressed as follows:

$$
\begin{aligned}
& y_{2}=\sqrt{P_{p}} h_{12} x_{p}+\sqrt{P_{a}} h_{32, k} x_{s}+w_{2}, \\
& y_{4}=\sqrt{P_{p}} h_{14} x_{p}+\sqrt{P_{a}} h_{34, k} x_{s}+w_{4},
\end{aligned}
$$

where, $P_{a}$ is the transmit power of $\mathrm{ST}_{k}$ and $w_{i}$ represents the Gaussian noise with the power of $\sigma_{w}^{2}$ at terminal $i$.

To improve the performance of primary receiver PR, the signal of secondary user $x_{s}$ should be filtered out from $y_{2}$ using the energy concentration property of chirp signal in the FrFT domain. After removing the estimation of $x_{s}$ from the received signal with the assumption of perfect channel estimation, the remaining signal can be denoted as:

$$
y_{p m}=\sqrt{P_{p}} h_{12} x_{p}+\sqrt{P_{a}} h_{32, k}\left(x_{s}-\widetilde{x_{s}}\right)+w_{2},
$$

where, $\widetilde{x}_{s}$ represents the signal filtered out in the FrFT domain which can be expressed as $\widetilde{x}_{s}=(1-e) x_{s}$ and $e$ is the signal separation error. Thus the achievable data rate of PR can be expressed as:

$$
R_{p}=\log _{2}\left(1+\frac{P_{p}\left|h_{12}\right|^{2}}{e^{2} P_{a}\left|h_{32, k}\right|^{2}+\sigma_{w}^{2}}\right) .
$$

At the secondary receiver SR, we can also utilize FrFT domain filtering to obtain the estimation of the signal from secondary user $x_{s}$. The filtered out signal with perfect channel estimation can be denoted as: 


$$
y_{s m}=\sqrt{P_{a}} h_{34, k} \widetilde{x_{s}}+w_{4},
$$

Thus the achievable data rate of SR is:

$$
R_{s}=\log _{2}\left(1+\frac{(1-e)^{2} P_{a}\left|h_{34, k}\right|^{2}}{\sigma_{w}^{2}}\right) .
$$

In order to achieve the optimal performance of the proposed spectrum sharing protocol, the transmit power of $\mathrm{ST}_{k}$ $P_{a}$ needs to be optimized to maximize the achievable data rate of secondary system subject to the target data rate constraint of primary user. This optimization problem can be formulated as:

$$
\max R_{s}
$$

subject to

$$
\begin{gathered}
R_{p} \geq R_{p t} \\
0 \leq P_{a} \leq P_{s},
\end{gathered}
$$

where, $P_{s}$ is the maximum transmit power constraint of secondary user. Substituting (7) into (10), and considering the fact that $\mathrm{ST}_{k}$ can obtain the tolerable interference of primary user $I$ from the spectrum sharing admission message, we can deduce the following formulation:

$$
e^{2} P_{a}\left|h_{32, k}\right|^{2} \leq I=\frac{P_{p}\left|h_{12}\right|^{2}}{\rho_{1}}-\sigma_{w}^{2} .
$$

Finally, the optimal transmit power $P_{a}$ in the access mode can be calculated as follows:

$$
P_{a}=\min \left(\frac{I}{e^{2}\left|h_{32, k}\right|^{2}}, P_{s}\right) \text {. }
$$

Thus, the achievable data rate of SR $R_{s}$ in Access Mode can be expressed as:

$$
R_{s}=\log _{2}\left(1+\frac{(1-e)^{2}\left|h_{34, k}\right|^{2}\left(\min \left(I /\left(e^{2}\left|h_{32, k}\right|^{2}\right), P_{s}\right)\right)}{\sigma_{w}^{2}}\right) .
$$

Assistance Mode: When $r_{12}<\rho_{1}$, i.e. the direct transmission link is too difficult to guarantee its requirement of target data rate and the primary system needs cooperation, primary receiver PR sends assistance request message which indicates the target cooperation gain $Q$. Upon hearing such message, $\mathrm{ST}_{i}$ calculates the priority value with the consideration of the channel conditions of $\mathrm{PT} \rightarrow \mathrm{ST}_{i}$ links $\left|h_{13, i}\right|_{2}^{2}, \mathrm{ST}_{i} \rightarrow \mathrm{PR}$ links $\left|h_{32, i}\right|^{2}$ and $\mathrm{ST}_{i} \rightarrow \mathrm{SR}$ links $\left|h_{34, i}\right|^{2}(i=1 \sim N)$, respectively.

The priority of each secondary user can be expressed as:

$$
\begin{aligned}
& M_{i}=u\left(\left|h_{13, i}\right|^{2}-\rho_{2}\right) u\left(\left|h_{32, i}\right|^{2}-\frac{Q \sigma_{w}^{2}}{P_{s}}\right)\left|h_{34, i}\right|^{2} \exp \left(\left|h_{32, i}\right|^{2}\right), \\
& i=1,2 \ldots N
\end{aligned}
$$

where, $u(x)$ is the step function and $\rho_{2}=2^{2 R_{p t}}-1$. When the channel gain of $\mathrm{PT} \rightarrow \mathrm{ST}_{i}$ links is higher than $\rho_{2}, \mathrm{ST}_{i}$ can decode the received signal from $\mathrm{PT}$ successfully and DF mechanism can be adopted to relay the primary signal. Moreover, when the channel gain of $\mathrm{ST}_{i} \rightarrow \mathrm{PR}$ link is higher than $Q \sigma_{w}^{2} / P_{s}, \mathrm{ST}_{i}$ is able to assist the primary user to achieve its target data rate. Otherwise, primary user cannot achieve its target data rate even though $\mathrm{ST}_{i}$ uses the total power to forward the primary signal.

From (14), it can be seen that the priority of secondary user increases rapidly with the channel gain of ST $\rightarrow$ PR link, and hence, secondary user with better channel condition of $\mathrm{ST} \rightarrow$ PR link is easier to be selected to assist primary user and higher cooperation gain can be obtained. Moreover, the priority of secondary user also increases with the channel gain of ST $\rightarrow$ SR link. Hence, the achievable data rate of secondary system can be improved.

Then, all the secondary users with $M_{i} \neq 0$ will start a count-down timer with the same value as in Access Mode. Assuming that the first timer reaching zero is in $\mathrm{ST}_{k}, \mathrm{ST}_{k}$ broadcasts an assistance response message to identify its presence. When the primary system receives the first assistance response message, the primary system knows that its target data rate can be achieved through cooperation with the secondary system. Then he corresponding secondary user will be selected and will cooperate with the primary system with the following two-phase DF relay procedure.

In the assistance mode, one whole transmission procedure is divided into two phases. In the first phase, the signal $x_{p}$ from PT is transmitted, and the signals received by primary receiver PR and secondary transmitter $\mathrm{ST}_{k}$ are denoted as $y_{2}^{(1)}$ and $y_{3, k}^{(1)}$, respectively. Then, they can be expressed as follows:

$$
\begin{gathered}
y_{2}^{(1)}=\sqrt{P_{p}} h_{12} x_{p}+w_{12}, \\
y_{3, k}^{(1)}=\sqrt{P_{p}} h_{13, k} x_{p}+w_{13, k} .
\end{gathered}
$$

In the second phase, $\mathrm{ST}_{k}$ decodes the primary signal $x_{p}$ from the received signal $y_{3, k}^{(1)}$ and adopts DF relaying to relay the signal of primary user. Since not all the power of secondary user is allocated to transmit the relayed signal of primary user, the remaining power is used to transmit its own signal, and the transmitted signal from $\mathrm{ST}_{k}$ in the second phase $x^{(2)}$ can be denoted as the linear combination of the regenerated signal $x_{p}$ with power $\left(P_{s}-P_{a}\right)$ and its own signal $x_{s}$ with power $P_{a}$, i.e., $x^{(2)}=\left(\sqrt[s]{P_{s}-P_{a}}\right) x_{p}+\sqrt{P_{a}} x_{s}$. Then, at PR and SR, the received signal can be formulated as:

$$
\begin{aligned}
& y_{2}^{(2)}=\sqrt{P_{s}-P_{a}} h_{32, k} x_{p}+\sqrt{P_{a}} h_{32, k} x_{s}+w_{32, k}, \\
& y_{4}^{(2)}=\sqrt{P_{s}-P_{a}} h_{34, k} x_{p}+\sqrt{P_{a}} h_{34, k} x_{s}+w_{34, k} .
\end{aligned}
$$

The signal of secondary user $x_{s}$ is filtered out from $y_{2}^{(2)}$ using the energy concentration property of chirp signals in the FrFT domain and the remaining signal is:

$$
y_{p m}=\sqrt{P_{s}-P_{a}} h_{32, k} x_{p}+\sqrt{P_{a}} h_{32, k}\left(x_{s}-\widetilde{x_{s}}\right)+w_{32, k} .
$$

Then combining the received signals $y_{2}^{(1)}$ and $y_{p m}$ using Maximal Ratio Combination (MRC) to decode $x_{p}$ at PR, the achievable data rate of $P R$ is:

$$
R_{p}=\frac{1}{2} \log _{2}\left(1+\frac{P_{p}\left|h_{12}\right|^{2}}{\sigma_{w}^{2}}+\frac{\left(P_{s}-P_{a}\right)\left|h_{32, k}\right|^{2}}{e^{2} P_{a}\left|h_{32, k}\right|^{2}+\sigma_{w}^{2}}\right) .
$$

After filtering out and decoding the secondary signal $x_{s}$, the achievable data rate of SR can be expressed as: 


$$
R_{s}=\frac{1}{2} \log _{2}\left(1+\frac{(1-e)^{2} P_{a}\left|h_{34, k}\right|^{2}}{\sigma_{w}^{2}}\right) .
$$

The optimization problem of power allocation in the assistance mode can also be denoted as (10). Since $\mathrm{ST}_{k}$ can obtain the knowledge of the cooperation gain the primary system needs from assistance request message, after substituting (20) and (21) into (10), we can deduce the following formulation:

$$
\frac{\left(P_{s}-P_{a}\right)\left|h_{32, k}\right|^{2}}{e^{2} P_{a}\left|h_{32, k}\right|^{2}+\sigma_{w}^{2}} \geq Q=\rho_{2}-\frac{P_{p}\left|h_{12}\right|^{2}}{\sigma_{w}^{2}} .
$$

Finally, the optimal power $P_{a}$ in the assistance mode can be obtained as follows:

$$
P_{a}=\frac{P_{s}\left|h_{32, k}\right|^{2}-Q \sigma_{w}^{2}}{\left|h_{32, k}\right|^{2}+Q e^{2}\left|h_{32, k}\right|^{2}} .
$$

Thus, the achievable data rate of $\mathrm{SR} R_{s}$ in Assistance Mode can be expressed as:

$$
R_{s}=\frac{1}{2} \log _{2}\left(1+\frac{(1-e)^{2}\left|h_{34, k}\right|^{2}}{\sigma_{w}^{2}}\left(\frac{P_{s}\left|h_{32, k}\right|^{2}-Q \sigma_{w}^{2}}{\left|h_{32, k}\right|^{2}+Q e^{2}\left|h_{32, k}\right|^{2}}\right)\right) \text {. }
$$

If none of the secondary user can help the primary user to achieve the target data rate, i.e., no ST responds to the assistance request message, the primary system will perform direct transmission mode without the assistance of secondary system.

In our proposed cooperative spectrum sharing protocol, the interference to and from the primary system can be greatly reduced thanks to the energy concentration property of chirp signals in the FrFT domain. Moreover, our proposed spectrum sharing protocol can be performed under different channel conditions of direct transmission link. Hence, spectrum resource can be fully utilized and outage probability of both primary and secondary system can be improved effectively. In addition, the joint optimization of power allocation and user selection of distributed secondary users is performed to reduce the interference to primary system and achieve a better performance of secondary achievable data rate.

\section{Performance Analysis}

To simplify the simulations, a round single-cell scenario with the normalized diameter of $d$ is considered as given in [8], where, the distance between PT and PR is normalized to " 1 ", and PT and PR are located at points $(-d / 2,0)$ and $(d / 2,0)$, respectively. STs are randomly distributed in the cell and SR is located at $(0, d / 2)$. The main configuration parameters are summarized in Table I.

\section{TABLE I SYSTEM PARAMETERS}

\begin{tabular}{ccc}
\hline Parameter & Symbol & Setting \\
\hline Cell Diameter & $d$ & 1 \\
Path Loss Exponent & $v$ & 4 \\
Signal Separation Error & $e$ & 0.1 \\
Primary Target Rate & $R_{p t}(\mathrm{bit} / \mathrm{s} / \mathrm{Hz})$ & 1 \\
Secondary Target Rate & $R_{s t}(\mathrm{bit} / \mathrm{s} / \mathrm{Hz})$ & 1 \\
\hline
\end{tabular}

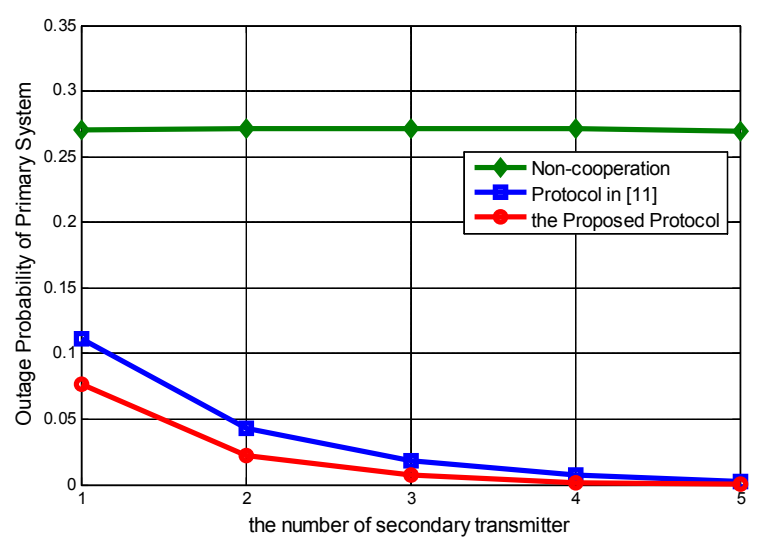

Fig.4 Outage probability of primary system vs. the number of secondary transmitter

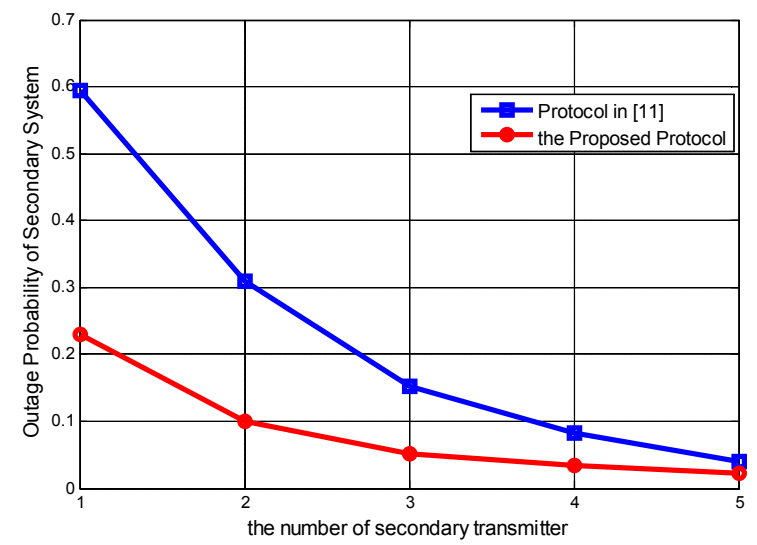

Fig.5 Outage probability of secondary system vs. the number of secondary transmitter

Fig.4 and Fig.5 compare the outage probability of primary and secondary system with our proposed protocol and the spectrum sharing protocol in [11], respectively, with $P_{p} / \sigma_{w}^{2}=P_{s} / \sigma_{w}^{2}=5 \mathrm{~dB}$. From Fig. 4 , it can be seen that the outage probability of primary system can be reduced to zero when the number of secondary transmitter $N>=4$ which indicates that the performance of primary system can be effectively improved through cooperation, and the outage probability for our proposed protocol is smaller than the protocol in [11]. From Fig.5 we can observe that the outage probability of the proposed protocol is reduced significantly for secondary system when comparing with the protocol in [11]. The reduction of outage probability is owing to the fact that the secondary signal can be filtered out from the primary signal through fractional Fourier domain filtering and the interference to both primary and secondary system can be greatly reduced. Moreover, from Fig. 4 and Fig. 5, we can also conclude that with the increase of secondary user number, the outage probability can be reduced, and the number of secondary user has more impact on the outage probability of secondary user. 


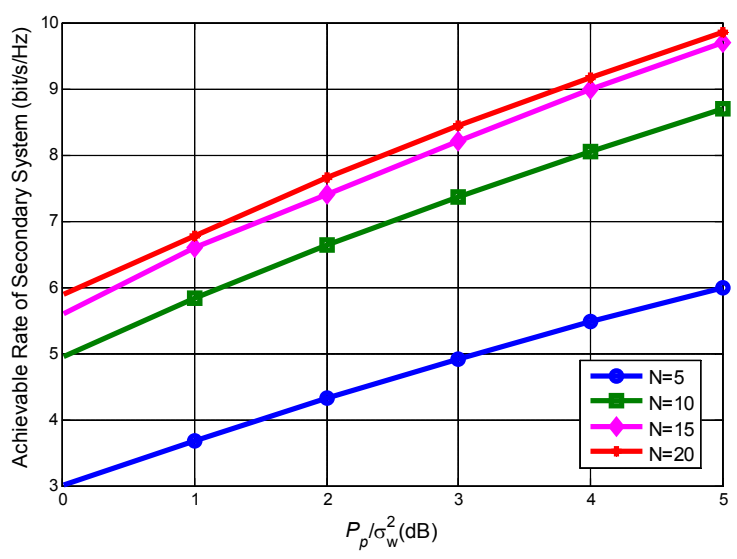

Fig.6 Achievable data rate of secondary system vs. $P_{p} / \sigma_{w}^{2}$

The performance of achievable data rate of secondary user is shown in Fig.6. It is obvious that with the increase of $P_{p} / \sigma_{w}^{2}$ and the number of secondary users $N$, the achievable data rate of secondary user can be improved significantly. It is because that secondary user can obtain more opportunity to access the spectrum in our proposed spectrum sharing protocol whatever the channel condition of direct transmission link is. In addition, when the number of secondary uses increases to 20 , it will be difficult for secondary system to achieve a better communication performance with a further increase in the secondary uses number.

\section{CONClusion}

In this paper, we propose a cooperative spectrum sharing protocol which employs transform domain processing technology. In our proposed protocol, primary user and secondary user which adopt sinusoidal signal and chirp signal, respectively, can be easily seperated. Hence, interference to primary system can be greatly reduced. Moreover, secondary users can access the spectrum or act as a DF relay to assist the system adaptively according to the channel conditions of primary direct transmission link which makes the spectrum sharing more flexible and improve the outage probability of secondary system effectively. Then we study the joint optimization of power allocation and user selection so that the transmission data rate of secondary user can be maximized on the premise that the primary user's data rate requirement is satisfied. Compared with the protocol in [11], the performance of outage probability of our proposed protocol is improved for both primary and secondary system and the improvement for secondary system is particularly remarkable. Moreover, achievable data rate of secondary system is also significantly improved with the increase of $P_{p} / \sigma_{w}^{2}$ and the number of secondary users.

\section{ACKNOWLEDGMENT}

This work has been supported in part by the National Basic Research Program of China (973 Program), under grant No.
2013CB329003, and National Nature Science Foundation under grant No. 61301100.

\section{REFERENCES}

[1]. A. G. Fragkiadakis, E. Z. Tragos and I. G. Askoxylakis, "A Survey on Security Threats and Detection Techniques in Cognitive Radio Networks," in IEEE Communications Surveys \& Tutorials, vol. 15, no. 1, pp. 428-445, First Quarter 2013.

[2]. L. Musavian and S. Aissa, "Capacity and power allocation for spectrum-sharing communications in fading channels," in IEEE Transactions on Wireless Communications, vol. 8, no. 1, pp. 148-156, Jan. 2009.

[3]. H. A. Bany Salameh, M. Krunz and O. Younis, "Cooperative Adaptive Spectrum Sharing in Cognitive Radio Networks," in IEEE/ACM Transactions on Networking, vol. 18, no. 4, pp. 1181-1194, Aug. 2010.

[4]. Y. Han, A. Pandharipande and S. H. Ting, "Cooperative spectrum sharing via controlled amplify-and-forward relaying," 2008 IEEE 19th International Symposium on Personal, Indoor and Mobile Radio Communications, Cannes, 2008, pp. 1-5.

[5]. Y. Han, A. Pandharipande and S. H. Ting, "Cooperative decode-and-forward relaying for secondary spectrum access," in IEEE Transactions on Wireless Communications, vol. 8 , no. 10, pp. 4945-4950, October 2009.

[6]. E. H. Shin and D. Kim, "Time and Power Allocation for Collaborative Primary-Secondary Transmission Using Superposition Coding," in IEEE Communications Letters, vol. 15, no. 2, pp. 196-198, February 2011.

[7]. W. Lu and J. Wang, "Opportunistic Spectrum Sharing Based on Full-Duplex Cooperative OFDM Relaying," in IEEE Communications Letters, vol. 18, no. 2, pp. 241244, February 2014.

[8]. W. Lu, J. Wang, W. Ge, F. Li, J. Hua and L. Meng, "An anti-interference cooperative spectrum sharing strategy with joint optimization of time and bandwidth," in Journal of Communications and Networks, vol. 16, no. 2, pp. 140-145, April 2014.

[9]. F. S. Tseng and C. Y. Hsu, "Robust Multiple-Antenna Cooperative Spectrum Sharing Design With Random Vector Quantization," in IEEE Transactions on Vehicular Technology, vol. 63, no. 7, pp. 3232-3249, Sept. 2014.

[10]. X. Wu, N. Fu and F. Labeau, "Relay-Based Cooperative Spectrum Sensing Framework Under Imperfect Channel Estimation," in IEEE Communications Letters, vol. 19, no. 2, pp. 239-242, Feb. 2015.

[11]. Y. Han, S. H. Ting and A. Pandharipande, "Cooperative Spectrum Sharing Protocol with Selective Relaying System," in IEEE Transactions on Communications, vol. 60, no. 1, pp. 62-67, January 2012.

[12]. A. Hyadi, El Mahdi Driouch, W. Ajib and M. S. Alouini, "Overlay cognitive radio systems with adaptive two-way relaying," 2013 IEEE Global Communications Conference (GLOBECOM), Atlanta, GA, 2013, pp. 937942. 